mages, et, tenant le principe de la responsabılıté, faire à la commune concluante toutes réserves pour fixer ultérieurement le montant des dommages qu'un nouveau traité avec un nouveau concessionnarre pourra infliger à la commune, et en prononcer la condamnation contre la Société :

Vu les conclusions déposíes par Me Bourguet, avoué, au nom de la Société du Sud-Electrique, tendant à ce qu'il plaise au Conseil dire que la commune ayant signé un cahier des charges dans lequel elle n'a prévu aucune indemnité, soit pour le retard, soit pour la dechéance, elle ne saurait aujourd'hui demander une indemnité, rejeter la demande de la commune;

Vu le traité passé entre la commune de Saint-Bauzille-er-Putois, et la Société du Sud-Electrique;

Vu ensemble les pièces du dossier ;

Vu les lois des 28 pluviôse, an VIII, 22 juillet 1889 ;

Ouı, Mc Caladoux, conseiller-rapporteur ;

Oui, Me Guibal, avocat, au nom de la commune de Saint-Bauzillecn-Putois, Me Bougault, avocat du barreau de Lyon, au nom de la Société du Sud-Electrique, et leurs observations orales;

Ou, M. le Commissaire du Gouvernement en ses conclusions,

Après en avoir délibéré conformément à la loi :

Considérant que la commune de Saint-Bauzille-en-Putors, au prétexte que la Société du Sud-Flectrique, qui a été substıtuce à la Société d'Applications industrielles, n’a pas tenu les obligatıons qui luı ont été imposées par le traité du vingt et un avril mll neuf cent cınq, demande qu'un délas soit imparti à ladite Société pour commencer les travaux nécessaires à l'éclalıage électrıque, sous peine de voir prononcer la résiliation du traté et des dommages-ıntérêts:

Considérant qu un contrat de concession constitue une convention d'un caractère spécıal, et que les regles qui le régissent ne se trouvent $\mathrm{n}$ i dans les textes de lois, ni dans des règlements, mais dans des conventions passées avec les concessionnaires, que, par suife. le cahier des charges doit être la loi des parties.

Consid́rant que le traité du vingt et un avril mil neuf cent cinq. intervenu entre la commune de Saint-Bauzille-en-Putors et la Société du Sud-Electrique n’a prévu aucune indemnité, soit pour le retard, soit pour la déchéance, que, seulement aux termes de l'art. 33 dudit traité, le concessionnaire peut encourır la déchéance pour navoir pas commencé les travaux dans le délai d'un an, à dater des autorisatıons préfectorales, que ce tratté ne stipule aucune autre pénalité, que, par suite, il y a lieu de faire droit à la demande de résiliation formée par la commune de Saint-Bauzille-en-Putois et de rejeter le surplus de sa demande, et de mettre les dépens à la charge de ladite Société,

Arrête :

Est résilié le tralté pour la fourniture de l'éclairage et de la force motrice p.ır l'énergie électrique, intervenu entre la commune de Saint-Bauzille-en-Putois et la Société du Sud-Electrique. La Société supportera les frais de l'instance.

Délibéré au Conseil, le deux juillet mil neuf cent neuf, et prononcé en audience publique de ce jour, à Montpellier, le dix du même mois, où étaient présents: MM. Caldié, président; Caladou, Alby, conseillers; Mounier, commissaire du Gouvernement; Paul Goudas, secrétaire-gretfier.

\section{Projet de loi relatif aux usines hydrauliques établies sur les cours d'eau navigables et les canaux}

happort présenté à la Chambre de Commerce de Lyon, dans sa bédnce du 21 octobre 1909, par M. Jean ColgNıT, vice-prissident.

\section{Messieurs,}

J'ai l'honneur de vous rendre compte de l'examen que votre Commission de législation a fait du projet de loi relatif aux ismes hydrauliques établies sur les cours d'eau et canaux du domaine public, qui a été voté par la Chambre des Députés, dans sa séance du 16 juillet Igog $\left({ }^{*}\right)$.

La Chambre de commerce de Lyon a pris déjà deux délibérations, les 22 janvier 1903 et 13 septembre 1906 , sur le projet de loi relatif aux usines hydrauliques sur les cours d'eau non navigables, ni flottables. Il est regrettable que ce

(*) Vorr l. texte d" la lol dans La Honizle Blanche d'octohre 1909. projet de loi ne soit jamais venu à l'ordre du jour de la Chambre des Députés Tel qu'ıl a été rédigé définitivenent par la Commission de la Chambre, d'accord avec le Gouvernement, il tenart compte, dans une large mesurc, des desiderata formulés par notre Chambre. Son adoption, en permettant d'éviter le chantage des barreurs de chute, donnerait un nouvel essor à l'aménagement des chutes des pays montagneux.

Le projet de loi relatıf aux usines hydrauliques établies sur les cours d'eau navigables et canaux du domane public a eu un tour de faveur, et a été voté sans débat par la Chambre des Députés. 11 soulève, en effet, des questions beaucoup moins délicates, le caractère domanial de ces cours d'eaux rendent l'Etat propriétarie de tous les drorts de riveraineté é de propriété sur ces cours d'eau, et libre, par conséquent, de disposer comme 1 l l'entend des chutes d'eau que l'on peut créer sur eux. C'est donc un projet de lor en quelque sorte administratif, tendant simplement à imposer par la los certaines règles générales dans les décisions que l'admmistration sera appelée à prendre à cet égard

L'Etat faisant toutefois appel à l'industric privée pour l'aménagement et l'exploitation des chutes d'eau, il a paru opportun à votre Commission de législation, d'examiner lc régime qui sera imposé dorénavant à cette industrie.

Ce régime sera celui de la concession, avec retour à l'Etat de l'usine concédée, à l'exprration de la concession.

Actuellement, le régime est celui de l'autorisation ou permission. Cette autorisation est donnée à titre précaire, c'est-àdire que l'administration se réserve toujours le droit de reti. rer, sans indemnité, l'autorisation. Cette autorisation est donnée par les Préfets, s'il est constaté que la prise d'eau n'aura pas pour effet, eu égard au volume des cours d'eau, d'en altérer le régime, ou encore s'il s'agrit d'une autorisation temporaire, de deux ans au plus. Dans les autres cas, l'autorisation est donnée par un décret rendu, après enquête, sur l'avis du Consell d'Etat. La modification ou le retrait de l'autorisation ne peurent avorr licu que suivant les formes ci-dessus (art. 40 à 45 de la lói du 8 avril i 898 ).

Jusqu'à la loi de I 898 sur le régime des eaux, la jurisprudence du Conseil d'Etat était que le retrait d'autorisation sans indemnité ne pouvalt avoir lieu que pour une rasson d'intérêt public (navigation, agriculturc, commerce, industrie, salubrité). L'autorisation n'était jamais retirée dans un intérêt privé, par exemple, pour donner la jouissance de la chutc d'eau à un autre titularre, ce nouveau titulaire fût-il une commune. On conçoit qu'un tel régime de précarıté, malgré les traditions de brenveillance de l'administration, ait permis à des industriels d'établir des moulins ou des petites usines, nais 11 est insuffisant pour rassurer les énormes capitaux nécessaires à l'aménagement des grandes chutes d'eau, que l'industrie moderne électrique permet d'utiliser

Avant la lo1 de 1898 , le Pouvorr exécutif pouvait certainement donner des concessions, aucun texte législatif ne le lui tófendant; mais aucune ne le lui permettait expressément, ct 11 préférait s'en tenir à de simples autorisations.

Quand on a voulu établir, en I 892, aux portes de I.yon, une usine de 12.000 chevaux sur une dérivation du Rhône, l'usine de Jonage, les promoteurs de cette entreprise ont demandé une concession pour laquelle les Pouvoirs Publics ont rendu une loi spéciale, cette lo étant du reste nécessaire pour concéder une distribution d'énergie électrique, la loi du I 5 juin Igo6 sur cette distribution n'existant pas encore.

Aujourd'hui, avec les termes de la loi de 1898 , il scmble bien que, pour chaque concession, il faille une loi spéciale. Cet état de choses est de nature à entraver l'utilisation des forces motrices de nos fleuves, et votre Commission est unanime à penser que la loi proposée vient combler utilement une lacune.

Seulcment, pour que cette loi suscite des entreprises, il ne taut pas que les charges imposées à l'entrepreneur soient telles que tout capitaliste sérieux en soit écarté. Ton élémont 
csscntrel d'une concession étant sa duréc, il faut que cette durce solt assez grande pour que toutes les dépenses de premicr établissement solent entièrement amorties à l'expiration le la concession et qu'il y ait, en outre, des perspectives de bénéfices assez importants et assez durables pour compenser les risques de l'entreprise. La concession de l'usine de Jonage a une durée de 99 ans. Mass c'était la première usine de ce scnre en France, et on peut admettre une durée plus courte aujourd'hui qu'on connaît mieux les conditions d'exploitation de ces usines. Fort sagement, le projet de loi renonce à fixer unc durée uniforme pour toutes les concessions, et laisse au pouvorr concédant le soin de fixer cette durée dans le cahier des charges de chaque entreprise.

S'inspirant de ce qui a été fait pour les chemins de fer, il clécide que la concession sera donnée par une loi lorsque le canal de dérivation d'une usine aura plus de 20 kilomètres, ou que la puissance brute dont l'usine pourra disposer à l'étrage dépassera 15.000 kilowatts, et qu'elle sera donnée par un décret pour toutes les usinss inférieures Nous n'avons pas d'objection à cette répartıtion.

Il nous reste smplement à souharter que les pouvours concédants ne cèdent pas trop faclement à la tendance actuelle de ne donner que de courtes concessions, ce qui serait de nature à cnrayer complètement la mise en valeur de cette richesse nationale. Dans chaque cas partıculier, la durée de la concession devra être mise en regard des charges imposées aux concessionnares Il sera souvent dans l'intérêt public, d'augmenter ces charges en augmentant par compensation la durée.

Le projet de loi ne fixe pas ces charges, il se contente d'en déterminer le cadre L'article 9 du projet de lo1 vise parmi ces charges les mesures 1ntéressant la navigation ou le flottage, la protection contre les inondations, la salubrité publique, l'alimentation des populations riveraines, les nécessités de l'rrrigation, la conservation et la libre circulation du poisson, la protection des paysages, les réserves en eau ou en force stipulées au profit des services publics, les conditions de rachat.

Le même article 9 renvore également au cahier des charges le soin de fixer le montant du cautionnement, celui de la redevance due à l'Etat pour la prise d'eau et autres conditions financières de la concession, et enfin les tarifs maxima à percevoir pour la vente au public de l'énergie.

La longue énumération de ces charges montre que leur total peut facilement dépasser les produits palpables de l'entreprise si le pouvour concédant, en en faisant une juste appréciation, ne modère pas l'appétit de chacun des intérêts publics mis ainsi en avant

Votre Commission pense que les charges qu'on peut rendre le plus onéreuses pour l'entreprise sont les mesures intéressant ia navigation, les réserves en eau ou en force au profit des services publics, et la redevance due à l'Etat; elle estime qu'il est regrettable qu'en en posant principe, le projet de loi ne marque pas un ordre de préférence, et une limitation à certaines d'entre elles

Votre Commission vous propose d'émettre le vœu qu'il sort introduit, dans le projet de loi, que partout où la navigation pourra être établie, ce doit-être l'intérêt prédominant.

En effet, la redevance due à l'Etat, dont le principe est posé dans l'article 44 de la loi du 8 avril i 898 , et le taux fixé par le décret du I 3 juillet Igo6, n'est qu'un intérêt fiscal qui cloit être modéré si l'on veut favoriser l'aménagement des chutes. Pour les concessions de force motrice, le décret du I3 juillet I906 fixe la redevance au I/Io de la valeur locative de la force concédée. Ce taux devrait être visé comme un maximum par le projet de loi, et pouvoir être abaissé en considération d'autres charges qui seraient imposées au concessionnaire.

Les réserves en eau ou en force pour les services publics constituent un principe tres dangereux. En effet, avec les transports de force à grande distance, tous les services publics dans un rayon de plus en plus étendu peuvent invoquer unc réserve en leur fareur. $S_{1}$ on les écoule tous, la chutc sera entrèrement absorbée. Il faudra donc farre un choix entre eux. Auquel donnera-t-on la préférence?

La loi du i 5 juin 1906 sur les distributions d'énergie élcc trique a très bien vu cette difficulté. Elle a sagement décidé que les concessionnares de distribution publique ne peuvent être assujettis qu'à des avantages envers l'Etat ou les communes consistant en prix réduits d'abonnement, comme ccux qui seraient accordés aux services publics pour les foumtures équivalentes.

Nous demandons formellement que les réserves en eau ou en force prévues par le nouveau projet de loi ne le solent que lans cette limite. Une réserve gratuite est absolument dangereuse. D'abord, on sera toujours tenté d'en exagérer l'importance, quitte pour le service public qui l'aura réclamée, à n'cn pas user. Mais, même dans ce cas, une fors inscrite dans ld concession, elle restera une menace permanente pour le concessionnaire et l'empêchera d'utiliser toute la force aménagée.

Dans le projet de lor sur les usines hydraulıques établics sur les cours u'eau non navigables, déposé par le Gouvernement le I 2 juin 1906, les réserves d'énergie au profit des services publics sont limitées au quart des basses eaux. En outrc, ces services n'ont le droit de réclamer cette énergie que pendant Io ans. Passé ce déla1, le concessionnaire peut en disposer. Il faudrait au moins imposer cette limitation. Mais nous estimons que partout où l'amélioration de la navigation est possible, il vaut mieux làsser toute l'énergie au concessionnaire en lui imposant unc contribution plus élevée aux travaux en faveur de la navigation, et se contenter, pour les autres zervices publics, de la stipulation des prix réduits d'abonnement.

Les réserves cn eau en faveur de l'urrigation inscrites au propet de loi appellent aussi quelques observations.

S'il s'agit de maintenir des irrigations existantes, il ne peut y avoir aucune objection. Mais s'il s'agit d'irrigations futures, la réserve peut être 11 limitée, et sous prétexte d'irrigations que l'avenir ne verra peut-être jamais réaliser, on risque d'cmpêcher la naissance d'industries qui augmenterarent la richesse nationale. Il faudrait donc que le projet imposât dès maintcnant une limite. Dans le projet de loi relatif aux cours d'eau non navigables, le gouvernement prévoyart que, seules, auraient droit à une réserve d'eau les entreprises d'alimentation des agglomérations non ruveraines et des entreprises d'irrigation collectives, qui se traduiraient en projet déposé dans les deux mois du décret de concession et qui seraient exécutées dans un délai de trois ans

Votre Commission réclame les mêmes restrictions.

Du reste, en ce qui concerne la région du Midi de la France, le Ministère de l'Agriculture songe, avec raison, à établir les 1rrigations, au fur et à mesure des demandes des Agriculteurs, au moyen d'usines de pompage mues par l'éncrgre électrique Ces usin's seront alors des services publics rentrant dans la catégorie de tous les services publics ayant drolt, non à de l'éner zie gratuite, mais à des prix de faveu' d'abonnement, comme nous l'avons exposé plus haut.

Les questions diverses d'intérêt public étant ainsi réglées, l'Etat aura à se préoccuper du plus important d'entre cux, puisqu'1l s'agit de cours d'eau navigables, c'est-à-dire de la navigation

A ce point de vue, le texte du projet de 101 aurait besoin d'être précisé lee paragraphe 5 de l'article 9 prévort, à titre de rederance, une contribution afférente à l'utilisation des ouvrages déjà établis par l'Etat dans l'intérêt de la navigation ou du flottage Mais là ne peut se borner la contribution au service de la navigation, etc'est dans le paragraphe 4 de cet article, prévoyant les mesures intéressant la navigation, que l'Administration pourrait puiser le droit d'imposer au concessionnaire l'exécution d'ouvrages nouveaux en faveur 
de la navigation. Nous pensons que c'est bien là l'interprétation à donner à cet article. Mais 1 l conviendraıt de le dire explicitement.

En effet, l'établissement de l'usine hydraulique est de nature à interrompre ou à gêner la navigation, soit que cette usine nécessite un barrage à travers la rivière, soit que par une dérivation elle prenne un volume d'eau ne laissant plus dans le lit de la rivière le tirant d'eau nécessaire. Il faudra, de toute nécessité, établir des écluses ou ascenseurs pour la navigation, dans le premier cas, et prévorr le canal de dérivation, en vue de la navigation, dans le second. Il se présentera deux hypothèses : ou bien les recettes de l'entreprise seront suffisantes $\mathrm{F}$ (ur rémunérer le capital nécessaire à ces travaux, et il sera juste de lui imposer cette dépense; ou bien clles ne le seront pas, et l'Etat aura à envisager si l'amélioration qui résultera, en général, pour la navigation de ces travaux, légitime qu'1l se contente d'une contribution partıelle. C'est la première hypothèse qui a été envisagée, lorsque l'Etat a imposé à la Compagnie de Jonage l'établissement d'écluses qui lui ont coûté 3.252.000 francs.

Le calcul était juste, puisque malgré cettc charge la Soclété est prospère. Cette combinaison peut donc être renouvelée. Nous irons plus loin, et nous pensons que la contribution du concessionnarre peut s'étendre à d'autres travaux concernant la navigation que ceux immédiatement nécessités par la construction de l'usine. Puisque l'Etat concède un bien qui est le sien, 1 l peut parfaitement imposer une contribution supplémentaire, pourvu que les conditions économiques de l'entreprise le comportent. L'Etat peut établir cette contribution supplémentaire, soit en abrégeant la durée de la concession, solt en augmentant la redevance en argent, ou les diverses réserves prévues. Mais aucune de ces mesures ne sera d'un intérêt comparable à celui que présentera l'amélioration de la navigation sur une section étendue d'un fleuve. ou d'une rivière.

On sait qu'il y a une demande de concession d'une usine hydraulique sur le Rhône, à Génissiat, près de Bellegardc Nin). Cette usine aurait une pussance de 75.000 kllowatts à l'étrage. Même après le vote du projet de loi que nous examinons, cette concession, vu son importance, nécessitera le vote d'une lo1 spéciale. Toutes les considérations que nous venons de farre valoir, au sujet de la navigation, auront là une application des plus intéressantes. Mais 11 est indispensable que, dans un projet de loi qui veut fixer les principes dont devront s'inspirer les lois spéclales, on prévole toutes les combinaisons possibles permettan' d'aménager un cours d'eau au double point de vue de la force motrice ct de la navigation. Ainsi seront accrues les richesses du pays. La création des usines consommatrices de force motrice sera grandement facilitée si ces usines peuvent recevoir leurs matières premières par la navigation. Au point de vue économique, les deux intérêts sont solidaires. C'est une rasson de plus pour les licr financièrement.

$A$ côté de la question des grandes usines, le projet de loi règle la situation des petites usines.

11 définit les petites usines " usines disposant d'unc purssance brute en étrage d'au plus 200 kilowats et qui n'ont pas pour objet principal le commerce de l'énergie n. Cette définition vise surtout, comme on le voit, les petites usines, telles que moulins, papeteries, etc., consommant elles-mêmes la torce hydraulique qu'elles empruntent aux cours d'eaux navigables.

Pour ces usines, le projet de loi maintient le régime actuel de l'autorisatıon, qui reste précarre et révocable, mais limite l'effet de cette autorisation à une durée maxima de cinquante années. Cette innovation nous paraît très mauvaise. Jusqu'ici les permissions sont données sans délai fixe, et la limitation. des durées était introduite seulement dans le régime des concessions.
Cette limitation de durée ne se comprend pas pour des usines appartenant, le plus souvent, à des particuliers. C'est assez que la permission pusse être retirée dans un intérêt puJlic, mais quand il n'y a aucun intérêt public en Jeu, on ne comprend pas cette limitation. Le projet de loi prévoit bien que la permission peut être renouvelée. Mais si elle ne l'est pas, le permissionnarre est tenu, au choix de l'Administration, soit de rétablır les lieux dans l'état primitif, soit d'abandonner à l'Etat, sans indemnité, ceux de ses ouvrages qui sont établis sur le domaine public fluvial. La première hypothèse ne peut exıster que dans le cas où l'ouvrage hydraulique est devenu une gêne pour les intérêts publics Mais en vertu de la précarité, l'ouvrage peut être supprimé à tout moment. Alors, pourquor le prévorr à date fixe? Serait-ce parce que, actuellement, l'mtérêt public auquel on sacrifiera un intérêt privé doit êtrc solennellement constaté par le Conscil d'Etat, tandis qu'avec le projet de lo1, à l'expiration du délai, cet intérêt privé pourra être sacrifié à la volonté de l'Administration, sans qu'elle ait à donner de motıfs, ni à prouver la réalité de l'intérêt public'

Dans la seconde hypotlièse, l'Etat s'emparant sans indemnité d'un ouvrage hydraulique, en fera usage pour lui, ou substituera un nouveau permissionnarre à l'ancien. Voit-on un petit usinier, travaillant depuis cinquante ans dans une locil. inté, oü il aura apporté un élément de richesse industrielle, frustré de la force hydraulique qu'il aura aménagée de scu deniers, au profit d'un de ses concurrents, qu1 viendra à sil porte lui farre une concurrence avec l'arde de l'Etat

Votre Commission prcteste contre cette conception inique. Elle demande le maintien du régime de la permission pour les petites usines, tel qu'il fonctionneactuellement. Le décret du I4 novembre 1899 qu fixe la redevance due à l'Etat pour les diverses usines a prévu la revision de cette redevance au moins tous les trente ans Les droits de l'Etat sont donc entièrement sauvegardés.

S'inspirant de la lor du i 5 jum 1906 sur les distributions d'énergie électrique, votre Commission demande en outre que, comme l'a fait cette lo1, lc petit usinier ait le chonx entre le régime actucl de la permission, avec les inconrónxents de la précarité, et le régime nouveau de la concession, instıtué pour les grandes usines, et qui ne serait obligatore que porr ces dernières.

Pour les usines existantes, al y en a deux catégories, colles qui ont une existence légale, et celles qui sont sous le régime de la permission précaire et révocable.

Par usines ayant une existence légale, on entend non sculement les usines qu, comme celle de Jonage, ont été concédées régulièrement, mais encore les usines qui ont un tutre de propriété véritable. Ce sont les usines qui ont un acte de concression antérieur à l'édit de Moulins de 1500, qui a proclamó la domanıalité inaliénable des cours d'eau navigables; celles qui, conformément à l'article premier de cet édit, ont été alıénées, par exception, par le pourour royal, après 1560 , avec clause de rachat; celles enfin qui ont été vendues comme bienu nationaux sous la Révolution. La situation de ces usines a ćté défine de nouveau par l'article 4.5 do la loi du 8 avril j 898 , qui dispose que leur prise d'eau peut toujours être modifié nu supprimée par l'Administration, mass moyennant indemnité.

L'article it du projet de loi déclare que la loi nouvelle ne leur sera pas appiricable.

Mais pour les usines existantes sous le régrme de la pormission, les articles 4 et 13 les assujettissent à. la nouvelle lor dans les conditions suivantes : les petites usine; celles inférieures à 200 kilowatts) voient la durée de leur prmission, actuellement indéfine, limitée suivant le principe du nouvoau régime. On leur concède seulement, dès maintenant, le max. mum de la durée prévue au nouveau régime, c'est-ì-dire cinquante années. Les grandes usines (celles supéricures à 200 ) kilowatts) ou même les petites, lorsqu'elles ont pour objet principal le commerce de l'énergie, scront, dans un dólai de 
cinc ans, placées sous le régine des usines cuncédées. Il suffira seulement d'un décret, quel que soit leur importance, pour leur donner cette concession.

Votre Commission proteste contre une modification sans indemnité des droits acquis

Elle a falt ressortir l'inconvénient pour les petites usines à venir, de la limitation de la durée des permissions. Pour les usines' existantes, construites sous le régme actuel, c'est une injustice de limiter après coup la durée de la permission et ce n'est pas parce que le projet de lo1 ajourne à cinquante ans la possibilité d'une spolration qu'il est plus acceptable.

Quant aux usines existantes que le projet oblige dans les cinq années à se transformer en usines concessionnaires, la méconnaissance des droits acquis est encore plus grave. Non seulement, on limite la durée de la permission comme dans le cas des petites usines, mais encore on impose toutes les charges du régime de la concession. Ces charges sont à la discrétion de l'administration, et on a vu qu'elles peuvent être considérables.

Votre Commission demande donc, pour les usines existantes, le maintien du statu quo, avec faculté néanmoins pour les usiniers de se ranger sous le régime de la concession, s'ils y trouvent avantage.

Votre Commission crolt, au contraire, nécessaire de corriger la rigueur de l'article 45 de la loi de 1898 qui refuse toute indemnité au permissionnaire qui se volt retirer sa permission, donnée à titre précaire Le législateur, en effet, à cette époque, ne faisait que confirmer la jurisprudence, étant entendu que l'Administration ne révoqualt d'autorisation qu'en vue d'un intérêt public.

Mais aujourd'hui, avec le développement de l'industrie électrique et des transports de force, 1 l est de l'intérêt général qu'il se contitue de grandes usines sur les cours d'eau navigables, et pour y arriver, il faudra souvent supprimer les petites usines, au vorsinage des grandes.

L'Administration va-t-elle profiter de la précarité de la permission de la petite usine pour la supprimer sans indemnité, afin de pouvoir donner librement la concession à la grande usine, ou, avec le système du projet de loi, va-t-elle attendre cinquante ans pour le faire? Ce serait injuste dans le premier cas, et contraire à l'intérêt général dans le second. La solution est de stipuler dans la nouvelle loi que le concessionnaire d'une grande usine doit indemniser la petite usine dont sa concession do1t amener la suppression, ou lui restituer en énergie électrique l'équivalent de l'eau qui lui est enlevée.

En résumé, votre Commission vous propose de demander au Sénat de voter le projet de lor relatif aux usines hydrauliques établies sur les cours d'eau navigables, qui lui a été envoyé par la Chambre des députés, avec les modifications suivantes :

$I^{\circ}$ Les charges à imposer aux concessionnaires devront l'être surtout en faveur de l'amélioration de la navigation du cours d'eau considéré.

$2^{\circ}$ Les réserves en eau ou en force à faire au profit des services publics devront être limitées, comme elles le sont dans la loi du I 5 juin I906, sur les distributions électrıques et dans le projet de loi du Gouvernement sur les usines hydrauliques établies sur les cours d'eau non navigables.

$3^{\circ}$ Le régime de l'autorisation sera maintenu tel qu'il l'est actuellement, sans limitation de durée.

$4^{\circ}$ Les petites usines et les usines existantes auront le choix entre le maintien du régime de l'autorisation et le nouveau régime de concession

$5^{\circ} \mathrm{Si}$ la concession de grandes usines entraine la suppression de petites usines, le concessionnaire devra indemniser la petite usine $(*)$.

(*) A l'unanimití, la Chambre de Commerce de Lyon a adopté rapport, dans ses termes et conclusions, el l'i converti en dibibération.

\section{LE RÉGLLAGE deS GROUPES ÉlEGTROGÈNES}

\author{
(Suile et $f n^{*}$ )
}

Accélérateur différentiel. - Cet appareil donne une vitesse de mancuvre proportionnelle à la différence entre le couple moteur et le couple résistant. Il ne sert qu'à modifier la vitessedc manœuvre de l'organe qui règle l'admission du fluide moteur; il ne peut donc être employé que comme organe accessoire du régulateur électro-mécanique précité.

L'accélérateur différentiel s'applique plus spécialement au cas où il s'agit de maintenir constante la vitesse d'un groupe électrogène. Il a pour but d'éviter les oscillations, et consiste à faire dépendre la vitesse de commande de l'or. gane qui règle l'admission du fluide moteur, non seule-

Fig. 3. - Schéma des connexions de l'accélérateur différentiel.
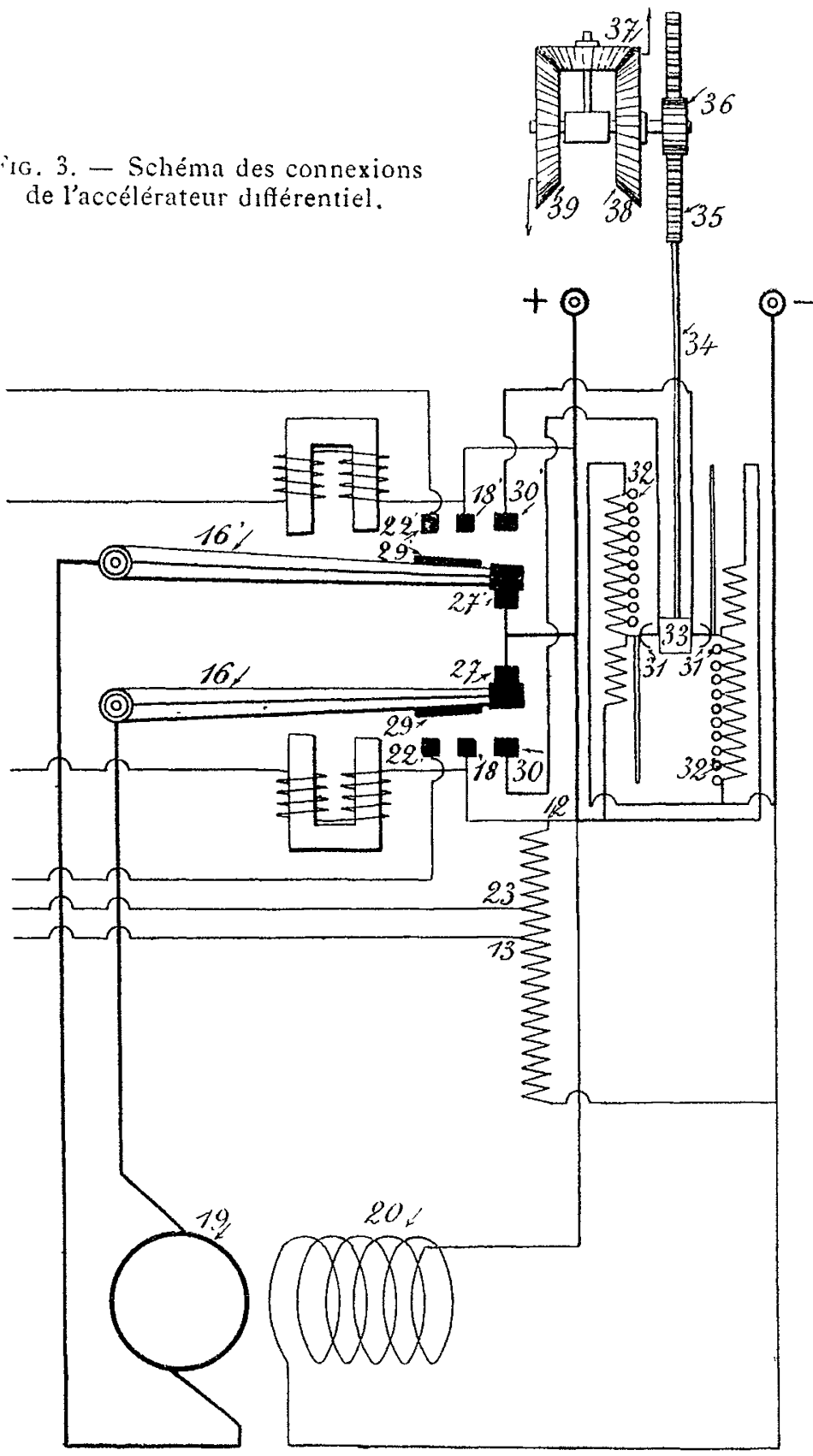

ment de la variation momentanée de la vitesse de rotation du groupe, mais encore de l'écart entre le couple moteur et le couple résistant, et ce, de façon que les conditions ciaprès soient remplies: 